\title{
Integrodifferential evolution systems with nonlocal initial conditions
}

Sylvain Koumla and Radu Precup

\begin{abstract}
The paper deals with systems of abstract integrodifferential equations subject to general nonlocal initial conditions. In order to allow the nonlinear terms of the equations to behave independently as much as possible, we use a vector approach based on matrices, vector-valued norms and a vector version of Krasnoselskii's fixed point theorem for a sum of two operators. The assumptions take into account the support of the nonlocal initial conditions and the hybrid character of the system. Two examples are given to illustrate the theory.
\end{abstract}

Mathematics Subject Classification (2010): 34K30, 35K90, 47J35.

Keywords: Integrodifferential equations, nonlinear evolution equation, nonlocal initial condition, delay, Krasnoselskii's fixed point theorem for a sum of operators.

\section{Introduction}

In this paper, we are concerned with the existence of solutions to the semilinear system of abstract integrodifferential equations with nonlocal initial conditions, of the type

$$
\left\{\begin{array}{l}
u_{i}^{\prime}(t)+A_{i} u_{i}(t)=\int_{0}^{t} K_{i}\left(t-s, u_{s}\right) d s+F_{i}\left(t, u_{t}\right), \quad t \in[0, T] \\
u_{i}(t)=\alpha_{i}(u)(t), \quad t \in[-\tau, 0], \quad i=1, \ldots, n .
\end{array}\right.
$$

Here $n \geq 1$, and for each $i \in I:=\{1, \ldots, n\}$, the linear operator $-A_{i}: D\left(A_{i}\right) \subseteq$ $X_{i} \rightarrow X_{i}$ generates a $C_{0}$-semigroup of contractions $\left\{S_{i}(t) ; t \geq 0\right\}$ on a Banach space $\left(X_{i},|\cdot|_{X_{i}}\right), \tau \geq 0, u \in C([-\tau, T], X)$, where $X=X_{1} \times \ldots \times X_{n}, u=\left(u_{1}, \ldots, u_{n}\right)$, and for each $t, u_{t}$ is the restriction of $u$ to $[t-\tau, t]$ shifted to the interval $[-\tau, 0]$, i.e., $u_{t} \in C([-\tau, 0], X)$ and

$$
u_{t}(s)=u(t+s), \quad s \in[-\tau, 0] .
$$


The nonlinear perturbations in equations are given by the continuous mappings $F_{i}$ from $[0, T] \times C([-\tau, 0], X)$ to $X_{i}, K_{i}$ from $[0, T] \times C([-\tau, 0], X)$ to $X_{i}$, and the nonlocal initial conditions are expressed by the continuous mappings $\alpha_{i}$ from $C([-\tau, T], X)$ to $C\left([-\tau, 0], X_{i}\right)$.

We note that the nonlocal initial conditions include in particular:

- the initial condition:

$$
u_{i}(t)=\varphi_{i}(t), \quad t \in[-\tau, 0], \quad i=1, \ldots, n
$$

where $\varphi=\left(\varphi_{1}, \ldots, \varphi_{n}\right) \in C([-\tau, 0], X)$ is given;

- linear multi-point conditions (linear nonlocal initial conditions of discrete type):

$$
u_{i}(t)=\varphi_{i}(t)+\sum_{j=1}^{m_{i}} a_{i j}(t) u_{i}\left(t+t_{i j}\right), \quad t \in[-\tau, 0], i=1, \ldots, n,
$$

where $0<t_{i j}<t_{i, j+1} \leq T$ for $j=1, \ldots, m_{i}$ and $i=1, \ldots, n$. The linear multipoint conditions include in particular the initial condition, and the periodicity condition

$$
u_{i}(t)=u_{i}(T+t), \quad t \in[-\tau, 0], \quad i=1, \ldots, n ;
$$

- linear nonlocal initial conditions of continuous type, given by integrals:

$$
\begin{aligned}
u_{i}(t) & =\varphi_{i}(t)+\int_{0}^{T} k_{i}(t, s) u_{i}(t+s) d s \\
& =\varphi_{i}(t)+\int_{t}^{T+t} k_{i}(t, s-t) u_{i}(s) d s, \quad t \in[-\tau, 0], \quad i=1, \ldots, n .
\end{aligned}
$$

Starting with Volterra's pioneering works on integrodifferential equations with delayed effects in population dynamics and materials with memory, the theory of delay differential equations has progressed continuously following the development of functional analysis and being stimulated by numerous applications in physics, chemistry, biology, medicine, economy, etc., see e.g., [23]), aimed to described evolution processes whose future states depend not only on the present, but also on the past history.

As concerns differential equations with nonlocal initial conditions of multi-point or integral type, we mention as some pioneering contributions, the papers of Cioranescu [15], Whyburn [42] and Conti [16]). Among further developments, we refer the readers to the works [2], [3], [7], [17], [21], [28], [29], [41], to the recent survey paper [35], and the references therein.

Parabolic problems with nonlocal initial conditions were considered in the papers of Kerefov [22], Vabishchevich [36], Chabrowski [14], Pao [33], Olmstead and Roberts [31], and Chapter 10 in [26], as nonlocal versions of some deterministic models from physics, mechanics, biology and medicine. Abstract evolution equations with nonlocal initial conditions were considered by Byszewski [11], Jackson [20], Lin and Liu [24]. For more recent contributions, we refer the readers to the papers [4], [6], [8], [10], [12], [19], [24], [25], [27], [30], [32], [39] and the recent monograph [9]. 
This paper has a double motivation. First, it is motivated by the second author's recent paper [5], which mainly inspires the operator technique of proof, and secondly, by the paper of Webb [40] for the class of integrodifferential equations.

There are several aspects in the present paper which are mixed together requiring a laboured technique of proof and yielding to a very general result:

- The use of the notion of support of a nonlocal initial condition and of a corresponding split norm. Throughout the paper, by $\left[-\tau, T_{0}\right]$ we shall denote the support of the nonlocal initial condition, that is the smallest subinterval $\left[-\tau, T_{0}\right]$ of $[-\tau, T]$ with $T_{0} \geq 0$ such that

$$
\alpha_{i}(u)=\alpha_{i}(v), i=1, \ldots, n, \text { for every } u, v \in C([-\tau, T], X)
$$

with $\left.u\right|_{\left[-\tau, T_{0}\right]}=\left.v\right|_{\left[-\tau, T_{0}\right]}$.

Here by $\left.u\right|_{\left[-\tau, T_{0}\right]}$ we mean the restriction of the function $u$ to the interval $\left[-\tau, T_{0}\right]$. Physically, this means that the evolution of a process is subjected to some constraints until a given moment of time $T_{0}$, and becomes free of any constraints after that moment.

The notion of support of a nonlocal initial condition was first used in the papers [7] and [8], and used after in [29], [2], [12], [4], [5]. As explained in these papers, and as we shall see in the following, stronger conditions on nonlinearities have to be asked on the support subinterval, compared to those required on the rest of the interval. Mathematically, the integral equation equivalent to the nonlocal initial problem is of Fredholm type on the support interval, and of Volterra type on the rest of the interval. This makes useful to consider a split norm on the functional space where the problem is studied. Thus, in connection with the delay system (1.1) and with the support $\left[-\tau, T_{0}\right]$ of the nonlocal initial condition, on a space of the type $C([-\tau, T], E)$, where $\left(E,|\cdot|_{E}\right)$ is a Banach space, we shall consider the split norm

$$
|u|_{\tau}=\max \left\{|u|_{C\left(\left[-\tau, T_{0}\right], E\right)}, \quad|u|_{C_{\theta}\left(\left[T_{0}-\tau, T\right], E\right)}\right\},
$$

where $|u|_{C\left(\left[-\tau, T_{0}\right], E\right)}$ is the usual max norm

$$
|u|_{C\left(\left[-\tau, T_{0}\right], E\right)}=\max _{t \in\left[-\tau, T_{0}\right]}|u(t)|_{E},
$$

while for any $\theta>0,|u|_{C_{\theta}\left(\left[T_{0}-\tau, T\right], E\right)}$ is the Bielecki type norm on $C\left(\left[T_{0}-\tau, T\right], E\right)$,

$$
\begin{aligned}
|u|_{C_{\theta}\left(\left[T_{0}-\tau, T\right], E\right)} & =\max _{t \in\left[T_{0}, T\right]}\left(\left|u_{t}\right|_{C([-\tau, 0], E)} e^{-\theta\left(t-T_{0}\right)}\right) \\
& =\max _{t \in\left[T_{0}, T\right]}\left(|u|_{C([t-\tau, t], E)} e^{-\theta\left(t-T_{0}\right)}\right) .
\end{aligned}
$$

In particular, when there is no a delay, i.e., when $\tau=0$, the norm (1.4) reduces to the split norm previously considered in [7], [2], [28] and [29].

- The hybrid character of the system. The system is split into to subsystems: the first $m$ equations for which Lipschitz conditions are assumed to guarantee that the corresponding integral operators are contractive, and the last $n-m$ equations $(0 \leq m \leq n)$ for which only at most linear growth conditions are required on the 
nonlinear terms, but in return, the compactness of the semigroups of operators is assumed to insure the compactness of the integral operators. In this way the proof will be a perfect illustration of Krasnoselskii's fixed point theorem for a sum of a compact map and a contraction, more exactly of its vector version of Viorel [37].

- The presence of integral terms. There is not only the bounded delay in the equations of system (1.1), but also cumulative integral terms which bring into the equations the whole history of the process. Such kind of equations arise from mathematical modeling of many real processes with memory from physics, biology and economics. These cumulative terms play a special role in the split analysis on two intervals as discussed previously.

\section{Preliminaries}

For the treatment of systems we use the vector approach based on vector-valued metrics and norms, and matrices instead of constants.

Let us make the convention that the elements of $\mathbb{R}^{n}$ are seen as column vectors. By a vector-valued metric on a set $E$ we mean a mapping $d: E \times E \rightarrow \mathbb{R}_{+}^{n}$ such that $d(x, y)=0$ if and only if $x=y ; d(x, y)=d(y, x)$ for all $x, y \in E$ and $d(x, y) \leq$ $d(x, z)+d(z, y)$ for all $x, y, z \in E$. Here by $\leq$ we mean the natural componentwise order relation of $\mathbb{R}^{n}$, more exactly, if $r, s \in \mathbb{R}^{n}, r=\left(r_{1}, \ldots, r_{n}\right), s=\left(s_{1}, \ldots, s_{n}\right)$, then by $r \leq s$ one means that $r_{i} \leq s_{i}$ for $i=1, \ldots, n$. A set $E$ together with a vector-valued metric $d$ is called a generalized metric space. For such a space, the notions of Cauchy sequence, convergence, completeness, open and closed set, are similar to those in usual metric spaces.

Similarly, a vector-valued norm on a linear space $E$, is defined as being a mapping $\|\cdot\|: E \rightarrow \mathbb{R}_{+}^{n}$ with $\|x\|=0$ only for $x=0 ; \quad\|\lambda x\|=|\lambda|\|x\|$ for $x \in E, \lambda \in \mathbb{R}$, and $\|x+y\| \leq\|x\|+\|y\|$ for every $x, y \in E$. To any vector-valued norm $\|\cdot\|$ one can associate the vector-valued metric $d(x, y):=\|x-y\|$. A linear space $E$ endowed with a vector-valued norm $\|\cdot\|$ is called a generalized Banach space if $E$ is complete with respect to the associated vector-valued metric $d$.

If $(E, d)$ is a generalized metric space with $d$ taking values in $\mathbb{R}^{n}$, we say that a mapping $\Gamma: E \rightarrow E$ is a generalized contraction (in Perov's sense) if there exists a square matrix $M$ of size $n$ with nonnegative entries such that its powers $M^{k}$ tend to the zero matrix 0 as $k \rightarrow \infty$, and

$$
d(\Gamma(x), \Gamma(y)) \leq M d(x, y) \text { for all } x, y \in E .
$$

Such a matrix is said to be a Lipschitz matrix. Notice that for a matrix $M$ the property $M^{k} \rightarrow 0$ as $k \rightarrow \infty$ is equivalent to the fact that the spectral radius $\rho(M)$ of the matrix $M$ is less than one. The role of matrices with spectral radius less than one in the study of operator systems was pointed out in [34], in connection with several abstract principles from nonlinear functional analysis.

For generalized contractions, the following extension of Banach's contraction principle holds. 
Theorem 2.1 (Perov). If $(E, d)$ is a complete generalized metric space, then any generalized contraction $\Phi: E \rightarrow E$ with the Lipschitz matrix $M$ has a unique fixed point $x^{*}$, and

$$
d\left(\Phi^{k}(x), x^{*}\right) \leq M^{k}(J-M)^{-1} d(x, \Phi(x))
$$

for all $x \in E$ and $k \in \mathbb{N}$ (where $J$ stands for the identity matrix of the same size as $M)$.

In this paper we use the following generalization of Theorem 2.1, a vector version of Krasnoselskii's fixed point theorem for a sum of two operators, owed to Viorel [37].

Theorem 2.2. Let $(E,\|\cdot\|)$ be a generalized Banach space, $D \subset E$ a nonempty bounded closed convex set and $\Gamma: D \rightarrow E$ a mapping such that

(i). $\Gamma=\Phi+\Psi$ with $\Phi: D \rightarrow E$ a generalized contraction in Perov's sense, and $\Psi: D \rightarrow E$ a compact operator;

(ii). $\Phi(u)+\Psi(v) \in D$ for every $u, v \in D$.

Then $\Gamma$ has at least one fixed point in $D$.

The following obvious proposition will be used in the proof of the main result.

Proposition 2.3. (a) If $M \in \mathcal{M}_{n \times n}\left(\mathbb{R}_{+}\right)$is a matrix with $\rho(M)<1$, then $\rho(\widetilde{M})<1$ for every matrix $\widetilde{M} \in \mathcal{M}_{n \times n}\left(\mathbb{R}_{+}\right)$whose elements are close enough to the corresponding elements of $M$.

(b) If $M \in \mathcal{M}_{n \times n}\left(\mathbb{R}_{+}\right)$is a matrix with $\rho(M)<1$, then $\rho(\widehat{M})<1$ for every matrix $\widehat{M} \in \mathcal{M}_{n \times n}\left(\mathbb{R}_{+}\right)$such that $\widehat{M} \leq M$ componentwise.

We conclude this preliminary section by a result about the compactness of the solution operator associated to a non-homogenous evolution equation [1].

Lemma 2.4 (Baras-Hassan-Veron). Let $A: D(A) \subset E \rightarrow E$ be the generator of a compact $C_{0}$-semigroup $\{S(t) ; t \geq 0\}$. Then for every uniformly integrable family of functions $\mathcal{F} \subset L^{1}(0, T ; E)$, the set of functions

$$
\left\{\int_{0}^{t} S(t-s) f(s) d s: f \in \mathcal{F}\right\}
$$

is relatively compact in $C([0, T], E)$.

For other basic notions and results of semigroup theory we mention the books [13], [18] and [38]. 


\section{Main result}

Looking for mild solutions to the problem (1.1), with $u_{i} \in C\left([-\tau, T], X_{i}\right)$ for $i=1, \ldots, n$ we are led in a standard way to the following integral system

$$
\left\{\begin{aligned}
u_{i}(t) & =\alpha_{i}(u)(t), \quad t \in[-\tau, 0], \\
u_{i}(t) & =S_{i}(t) \alpha_{i}(u)(0)+\int_{0}^{t} S_{i}(t-s) \int_{0}^{s} K_{i}\left(s-\sigma, u_{\sigma}\right) d \sigma d s \\
& +\int_{0}^{t} S_{i}(t-s) F_{i}\left(s, u_{s}\right) d s, \quad t \in[0, T], \quad i=1, \ldots, n .
\end{aligned}\right.
$$

Our assumptions are given differently for two sets of indices,

$$
I_{1}:=\{1, \ldots, m\} \quad \text { and } \quad I_{2}:=\{m+1, \ldots, n\},
$$

where $0 \leq m \leq n$, and it is understood that $I_{1}=\emptyset$ if $m=0$, and $I_{2}=\emptyset$ if $m=n$. Let $p>1$ be any fixed number.

The hypotheses are:

$\left(\mathbf{H}_{0}\right)$ (a) For each $i \in I_{1}$, the linear operator $-A_{i}: D\left(A_{i}\right) \subset X_{i} \rightarrow X_{i}$ generates a $C_{0}$-semigroup of contractions on the Banach space $X_{i}$.

(b) For each $i \in I_{2}$, the linear operator $-A_{i}: D\left(A_{i}\right) \subset X_{i} \rightarrow X_{i}$ generates a compact $C_{0}$-semigroup of contractions on the Banach space $X_{i}$.

$\left(\mathbf{H}_{1}\right)$ (a) For each $i \in I_{1}, K_{i}:[0, T] \times C([-\tau, 0], X) \rightarrow X_{i}$, is continuous, and there exist $a_{i j} \in C\left([0, T], \mathbb{R}_{+}\right)$for $j \in I$, such that

$$
\left|K_{i}(t, u)-K_{i}(t, v)\right|_{X_{i}} \leq \sum_{j=1}^{n} a_{i j}(t)\left|u_{j}-v_{j}\right|_{C\left([-\tau, 0], X_{j}\right)}
$$

for all $u, v \in C([-\tau, 0], X)$ and $t \in[0, T]$.

(b) For each $i \in I_{2}, K_{i}:[0, T] \times C([-\tau, 0], X) \rightarrow X_{i}$, is continuous, and there exist $d_{i}, a_{i j} \in C\left([0, T], \mathbb{R}_{+}\right)$for all $j \in I$, such that

$$
\left|K_{i}(t, u)\right|_{X_{i}} \leq \sum_{j=1}^{n} a_{i j}(t)\left|u_{j}\right|_{C\left([-\tau, 0], X_{j}\right)}+d_{i}(t)
$$

for all $u \in C([-\tau, 0], X)$ and $t \in[0, T]$.

$\left(\mathbf{H}_{2}\right)$ (a) For each $i \in I_{1}, \quad F_{i}:[0, T] \times C([-\tau, 0], X) \rightarrow X_{i}$ is continuous and there exists $b_{i j} \in C\left([0, T], \mathbb{R}_{+}\right)$for all $j \in I$, such that

$$
\left|F_{i}(t, u)-F_{i}(t, v)\right|_{X_{i}} \leq \sum_{j=1}^{n} b_{i j}(t)\left|u_{j}-v_{j}\right|_{C\left([-\tau, 0], X_{j}\right)}
$$

for $u, v \in C([-\tau, 0], X)$ and $t \in[0, T]$.

(b) For each $i \in I_{2}, F_{i}:[0, T] \times C\left([-\tau, 0], X_{i}\right) \rightarrow X_{i}$ is continuous and there exist $f_{i}, b_{i j} \in C\left([0, T], \mathbb{R}_{+}\right)$for all $j \in I$, such that

$$
\left|F_{i}(t, u)\right|_{X_{i}} \leq \sum_{j=1}^{n} b_{i j}(t)\left|u_{j}\right|_{C\left([-\tau, 0], X_{j}\right)}+f_{i}(t)
$$

for all $u \in C([-\tau, 0], X)$ and $t \in[0, T]$. 
$\left(\mathbf{H}_{3}\right)$ For each $i \in I, \quad \alpha_{i}: C([-\tau, T], X) \rightarrow C\left([-\tau, 0], X_{i}\right)$ and there exist $c_{i j} \in \mathbb{R}_{+}$for all $j \in I$, such that

$$
\left|\alpha_{i}(u)-\alpha_{i}(v)\right|_{C\left([-\tau, 0], X_{i}\right)} \leq \sum_{j=1}^{n} c_{i j}\left|u_{j}-v_{j}\right|_{C\left(\left[-\tau, T_{0}\right], X_{j}\right)}
$$

for all $u, v \in C([-\tau, T], X)$.

Theorem 3.1. Assume that the conditions $\left(\mathrm{H}_{0}\right)-\left(\mathrm{H}_{3}\right)$ hold. In addition assume that the spectral radius of the $n \times n$ square matrix $M=\left[m_{i j}\right]$, where

$$
m_{i j}=T_{0}\left|a_{i j}\right|_{L^{1}\left(0, T_{0}\right)}+\bar{a}_{i j}+\left|b_{i j}\right|_{L^{1}\left(0, T_{0}\right)}+c_{i j} \quad \text { for } i, j \in I,
$$

and

$$
\bar{a}_{i j}=\int_{T_{0}}^{T} d \xi \int_{0}^{T_{0}} a_{i j}(\xi-\sigma) d \sigma
$$

is less than one.

Then the problem (1.1) has at least one mild solution $u \in C([-\tau, T], X)$. In case that $m=n$, the solution $u$ is unique.

Proof. The integral system (3.1) can be seen as a fixed point equation $u=\Gamma(u)$ in $C([-\tau, T], X)$ for the nonlinear operator $\Gamma$ from the space $C([-\tau, T], X)$ to itself, $\Gamma=\left(\Gamma_{1}, \ldots, \Gamma_{n}\right)$, where $\Gamma_{i}: C([-\tau, T], X) \rightarrow C\left([-\tau, T], X_{i}\right)$ are defined by

$$
\left\{\begin{aligned}
\Gamma_{i}(u)(t) & =\alpha_{i}(u)(t), \quad t \in[-\tau, 0], \\
\Gamma_{i}(u)(t) & =S_{i}(t) \alpha_{i}(u)(0)+\int_{0}^{t} S_{i}(t-s) \int_{0}^{s} K_{i}\left(s-\sigma, u_{\sigma}\right) d \sigma d s \\
& +\int_{0}^{t} S_{i}(t-s) F_{i}\left(s, u_{s}\right) d s, \quad t \in[0, T] .
\end{aligned}\right.
$$

Clearly, the operator $\Gamma$ admits the representation $\Gamma=\Phi+\Psi$, where

$$
\Phi=\left(\Gamma_{1}, \ldots, \Gamma_{m}, \Phi_{m+1}, \ldots, \Phi_{n}\right), \quad \Psi=\left(0, \ldots, 0, \Psi_{m+1}, \ldots, \Psi_{n}\right),
$$

where for $i \in J_{2}$,

$$
\Phi_{i}(u)(t)= \begin{cases}\alpha_{i}(u)(t), & t \in[-\tau, 0] \\ S_{i}(t) \alpha_{i}(u)(0), & t \in[0, T]\end{cases}
$$

and

$$
\Psi_{i}(u)(t)=\left\{\begin{array}{l}
0, t \in[-\tau, 0] \\
\int_{0}^{t} S_{i}(t-s) \int_{0}^{s} K_{i}\left(s-\sigma, u_{\sigma}\right) d \sigma d s+\int_{0}^{t} S_{i}(t-s) F_{i}\left(s, u_{s}\right) d s, \quad t \in[0, T] .
\end{array}\right.
$$

We shall apply the vector version of Krasnoselskii's fixed point theorem to the operator $\Gamma$ on the space

$$
E:=C([-\tau, T], X)=C\left([-\tau, T], X_{1}\right) \times \ldots \times C\left([-\tau, T], X_{n}\right)
$$

endowed with the vector-valued norm

$$
\|u\|=\left(\left|u_{1}\right|_{\tau}, \ldots,\left|u_{n}\right|_{\tau}\right)^{t r},
$$


where for each $i$, by $\left|u_{i}\right|_{\tau}$ we mean the norm in $C\left([-\tau, T], X_{i}\right)$ given by (1.4), with $\theta>0$ large enough chosen below, and to a bounded closed convex subset $D$ of the form

$$
\begin{aligned}
D & =\left\{u=\left(u_{1}, \ldots, u_{n}\right) \in C([-\tau, T], X):\left|u_{i}\right|_{\tau} \leq R_{i} \text { for } i \in I\right\} \\
& =\{u \in C([-\tau, T], X):\|u\| \leq R\}
\end{aligned}
$$

with conveniently chosen radii $R_{i}, i \in I$. Here the notation $R$ stands for the vector column $\left(R_{1}, \ldots, R_{n}\right)^{t r}$. The result will follow from Theorem 2.2 once the following lemmas have been proved:

Lemma 3.2. There exists $R \in \mathbb{R}_{+}^{n}$ such that $\|\Phi(u)+\Psi(v)\| \leq R$ for all $u, v$ $\in C([-\tau, T], X)$ satisfying $\|u\|,\|v\| \leq R$.

Lemma 3.3. The operator $\Phi$ is a generalized contraction in Perov's sense on $C([-\tau, T], X)$.

Lemma 3.4. The operator $\Psi$ is completely continuous on $C([-\tau, T], X)$.

Proof of Lemma 3.2. Let $R \in \mathbb{R}_{+}^{n}$. The result will follow once we have proved that

$$
\|\Phi(u)+\Psi(v)\| \leq \widetilde{M} R+\Lambda,
$$

for all $u, v \in C([-\tau, T], X)$ with $\|u\|,\|v\| \leq R$, and some vector $\Lambda \in \mathbb{R}_{+}^{n}$ and matrix $\widetilde{M}$ close enough $M$ such that $\rho(\widetilde{M})<1$. Indeed, in this case, we can find a vector $R \in \mathbb{R}_{+}^{n}$ such that

$$
\widetilde{M} R+\Lambda \leq R
$$

that is $(J-\widetilde{M}) R \geq \Lambda$, for example, the vector $R=(J-\widetilde{M})^{-1} \Lambda$. The vector $R$ belongs to $\mathbb{R}_{+}^{n}$ since the matrix $J-\widetilde{M}$ is inverse-positive as a consequence of the fact that $\rho(\widetilde{M})<1$ (see, e.g., [34]).

Thus, in order to obtain (3.4) we need estimates of the norms $\left|\Phi_{i}(u)+\Psi_{i}(v)\right|_{\tau}$. Clearly, $\Phi_{i}(u)+\Psi_{i}(v)=\Gamma_{i}(u)$ for $i \in I_{1}$.

First note that from $\left(H_{1}\right)(a)$, for $v=0$,

$$
\left|K_{i}(t, u)\right|_{X_{i}} \leq \sum_{j=1}^{n} a_{i j}(t)\left|u_{j}\right|_{C\left([-\tau, 0], X_{j}\right)}+\left|K_{i}(t, 0)\right|_{X_{i}},
$$

hence the inequality in $\left(H_{1}\right)(b)$ also holds for $i \in I_{1}$, with $d_{i}(t)=\left|K_{i}(t, 0)\right|_{X_{i}}$. Similarly, the inequality in $\left(H_{2}\right)(b)$ holds for $i \in I_{1}$ with $f_{i}=\left|F_{i}(0)\right|_{C\left([-\tau, 0], X_{i}\right)}$. Also, from $\left(\mathrm{H}_{3}\right)$, one has

$$
\left|\alpha_{i}(u)\right|_{C\left([-\tau, 0], X_{i}\right)} \leq \sum_{j=1}^{n} c_{i j}\left|u_{j}\right|_{C\left(\left[-\tau, T_{0}\right], X_{j}\right)}+h_{i}
$$


for all $i \in I$ with $h_{i}=\left|\alpha_{i}(0)\right|_{C\left([-\tau, 0], X_{i}\right)}$.

For $t \in[-\tau, 0]$, we have

$$
\begin{aligned}
\left|\alpha_{i}(u)(t)\right|_{X_{i}} & \leq\left|\alpha_{i}(u)\right|_{C\left([-\tau, 0], X_{i}\right)} \leq \sum_{j=1}^{n} c_{i j}\left|u_{j}\right|_{C\left(\left[-\tau, T_{0}\right], X_{j}\right)}+h_{i} \\
& \leq \sum_{j=1}^{n} c_{i j}\left|u_{j}\right|_{\tau}+h_{i} \leq \sum_{j=1}^{n} c_{i j} R_{j}+h_{i}
\end{aligned}
$$

For $t \in\left[0, T_{0}\right]$ and $i \in I_{1}$, since the semigroups are of contractions,

$$
\begin{aligned}
\left|\Gamma_{i}(u)(t)\right|_{X_{i}} \leq\left|\alpha_{i}(u)(0)\right|_{X_{i}} & +\int_{0}^{t} \int_{0}^{s}\left|K_{i}\left(s-\sigma, u_{\sigma}\right)\right|_{X_{i}} d \sigma d s \\
& +\int_{0}^{t}\left|F_{i}\left(s, u_{s}\right)\right|_{X_{i}} d s .
\end{aligned}
$$

From (3.5), the first term is estimated as above, that is

$$
\left|\alpha_{i}(u)(0)\right|_{X_{i}} \leq \sum_{j=1}^{n} c_{i j} R_{j}+h_{i}
$$

while the integrals are estimated as follows:

$$
\begin{aligned}
& \int_{0}^{t} \int_{0}^{s}\left|K_{i}\left(s-\sigma, u_{\sigma}\right)\right|_{X_{i}} d \sigma d s \\
\leq & \int_{0}^{t} \int_{0}^{s}\left(\sum_{j=1}^{n} a_{i j}(s-\sigma)\left|\left(u_{j}\right)_{\sigma}\right|_{C\left([-\tau, 0], X_{j}\right)}+d_{i}(s-\sigma)\right) d \sigma d s \\
= & \sum_{j=1}^{n} \int_{0}^{t} \int_{0}^{s} a_{i j}(s-\sigma)\left|u_{j}\right|_{C\left([\sigma-\tau, \sigma], X_{j}\right)} d \sigma d s+T_{0}\left|d_{i}\right|_{L^{1}\left(0, T_{0}\right)}
\end{aligned}
$$

and

$$
\begin{aligned}
\int_{0}^{t}\left|F_{i}\left(s, u_{s}\right)\right|_{X_{i}} d s & \leq \int_{0}^{t}\left(\sum_{j=1}^{n} b_{i j}(s)\left|\left(u_{j}\right)_{s}\right|_{C\left([-\tau, 0], X_{j}\right)}+f_{i}(s)\right) d s \\
& =\sum_{j=1}^{n} \int_{0}^{t} b_{i j}(s)\left|u_{j}\right|_{C\left([s-\tau, s], X_{j}\right)} d s+T_{0}\left|f_{i}\right|_{L^{1}\left(0, T_{0}\right)} .
\end{aligned}
$$

Since $0 \leq s \leq t \leq T_{0}$, one has $\left|u_{j}\right|_{C\left([s-\tau, s], X_{j}\right)} \leq\left|u_{j}\right|_{C\left(\left[-\tau, T_{0}\right], X_{j}\right)} \leq\left|u_{j}\right|_{\tau}$. 
Then (3.8) and (3.9) give

$$
\begin{aligned}
\int_{0}^{t} \int_{0}^{s}\left|K_{i}\left(s-\sigma, u_{\sigma}\right)\right|_{X_{i}} d \sigma d s & \leq \sum_{j=1}^{n} \int_{0}^{t} \int_{0}^{s} a_{i j}(s-\sigma)\left|u_{j}\right|_{\tau} d \sigma d s+T_{0}\left|d_{i}\right|_{L^{1}\left(0, T_{0}\right)} \\
& =\sum_{j=1}^{n}\left|u_{j}\right|_{\tau} \int_{0}^{t} \int_{0}^{s} a_{i j}(s-\sigma) d \sigma d s+T_{0}\left|d_{i}\right|_{L^{1}\left(0, T_{0}\right)} \\
& \leq T_{0} \sum_{j=1}^{n}\left|a_{i j}\right|_{L^{1}\left(0, T_{0}\right)} R_{j}+T_{0}\left|d_{i}\right|_{L^{1}\left(0, T_{0}\right)}
\end{aligned}
$$

and

$$
\int_{0}^{t}\left|F_{i}\left(s, u_{s}\right)\right|_{X_{i}} d s \leq \sum_{j=1}^{n}\left|b_{i j}\right|_{L^{1}\left(0, T_{0}\right)} R_{j}+\left|f_{i}\right|_{L^{1}\left(0, T_{0}\right)} .
$$

Hence for $t \in\left[-\tau, T_{0}\right]$ and all $i \in I_{1}$, from (3.7), (3.10) and (3.11), we deduce that

$$
\begin{aligned}
\left|\Gamma_{i}(u)(t)\right|_{X_{i}} & \leq \sum_{j=1}^{n}\left(T_{0}\left|a_{i j}\right|_{L^{1}\left(0, T_{0}\right)}+\left|b_{i j}\right|_{L^{1}\left(0, T_{0}\right)}+c_{i j}\right)\left|u_{j}\right|_{\tau}+\lambda_{i} \\
& =\sum_{j=1}^{n}\left(m_{i j}-\bar{a}_{i j}\right)\left|u_{j}\right|_{\tau}+\lambda_{i}
\end{aligned}
$$

where $\lambda_{i}=T_{0}\left|d_{i}\right|_{L^{1}\left(0, T_{0}\right)}+\left|f_{i}\right|_{L^{1}\left(0, T_{0}\right)}+h_{i}$. Therefore

$$
\left|\Gamma_{i}(u)\right|_{C\left(\left[-\tau, T_{0}\right], X_{i}\right)} \leq \sum_{j=1}^{n}\left(m_{i j}-\bar{a}_{i j}\right)\left|u_{j}\right|_{\tau}+\lambda_{i}
$$

Next we estimate

$$
\left|\Gamma_{i}(u)\right|_{C_{\theta}\left(\left[T_{0}-\tau, T\right], X_{i}\right)}=\max _{t \in\left[T_{0}, T\right]}\left(\left|\Gamma_{i}(u)\right|_{C\left([t-\tau, t], X_{i}\right)} e^{-\theta\left(t-T_{0}\right)}\right)\left(i \in I_{1}\right) .
$$

To do this, take any $t \in\left[T_{0}, T\right]$ and $s \in[t-\tau, t]$. For $s \leq T_{0}$, we already have the estimate given by (3.13). Let $s \in\left[T_{0}, t\right]$. Then

$$
\begin{aligned}
& \Gamma_{i}(u)(s) \\
= & \Gamma_{i}(u)\left(T_{0}\right)+\int_{T_{0}}^{s} S_{i}(s-\xi) F_{i}\left(\xi, u_{\xi}\right) d \xi+\int_{T_{0}}^{s} S_{i}(s-\xi) \int_{0}^{\xi} K_{i}\left(\xi-\sigma, u_{\sigma}\right) d \sigma d \xi \\
= & \Gamma_{i}(u)\left(T_{0}\right)+\int_{T_{0}}^{s} S_{i}(s-\xi) F_{i}\left(\xi, u_{\xi}\right) d \xi+\int_{T_{0}}^{s} S_{i}(s-\xi) \int_{0}^{T_{0}} K_{i}\left(\xi-\sigma, u_{\sigma}\right) d \sigma d \xi \\
& +\int_{T_{0}}^{s} S_{i}(s-\xi) \int_{T_{0}}^{\xi} K_{i}\left(\xi-\sigma, u_{\sigma}\right) d \sigma d \xi .
\end{aligned}
$$

Using $\left(\mathrm{H}_{1}\right)(\mathrm{b})$, one has

$$
\left|\int_{T_{0}}^{s} S_{i}(s-\xi) \int_{0}^{T_{0}} K_{i}\left(\xi-\sigma, u_{\sigma}\right) d \sigma d \xi\right|_{X_{i}} \leq \sum_{j=1}^{n} \bar{a}_{i j}\left|u_{j}\right|_{\tau}+\left|f_{i}\right|_{L^{1}\left(0, T_{0}\right)},
$$


where

$$
\bar{a}_{i j}=\int_{T_{0}}^{T} d \xi \int_{0}^{T_{0}} a_{i j}(\xi-\sigma) d \sigma
$$

Furthermore

$$
\begin{aligned}
& \left|\int_{T_{0}}^{s} S_{i}(s-\xi) \int_{T_{0}}^{\xi} K_{i}\left(\xi-\sigma, u_{\sigma}\right) d \sigma d \xi\right|_{X_{i}} \\
& \leq \int_{T_{0}}^{s} \int_{T_{0}}^{\xi}\left(\sum_{j=1}^{n} a_{i j}(\xi-\sigma)\left|\left(u_{j}\right)_{\sigma}\right|_{C\left([-\tau, 0], Z_{j}\right)} e^{-\theta\left(\sigma-T_{0}\right)} e^{\theta\left(\sigma-T_{0}\right)}+d_{i}(\xi-\sigma)\right) d \sigma d \xi \\
& \leq \sum_{j=1}^{n}\left|u_{j}\right|_{\tau} \int_{T_{0}}^{s} \int_{T_{0}}^{\xi} a_{i j}(\xi-\sigma) e^{\theta\left(\sigma-T_{0}\right)} d \sigma d \xi+\left(T-T_{0}\right)\left|d_{i}\right|_{L^{1}\left(0, T-T_{0}\right)} .
\end{aligned}
$$

Next using Holder's inequality gives

$$
\begin{aligned}
\left|\int_{T_{0}}^{s} S_{i}(s-\xi) \int_{T_{0}}^{\xi} K_{i}\left(\xi-\sigma, u_{\sigma}\right) d \sigma d \xi\right|_{X_{i}} & \leq \frac{1}{\theta(q \theta)^{1 / q}} e^{\theta\left(t-T_{0}\right)} \sum_{j=1}^{n}\left|a_{i j}\right|_{L^{p}\left(0, T-T_{0}\right)}\left|u_{j}\right|_{\tau} \\
& +\left(T-T_{0}\right)\left|d_{i}\right|_{L^{1}\left(0, T-T_{0}\right)} .
\end{aligned}
$$

Similar arguments yield

$$
\left|\int_{T_{0}}^{s} S_{i}(s-\xi) F_{i}\left(\xi, u_{\xi}\right) d \xi\right|_{X_{i}} \leq \frac{1}{\theta} e^{\theta\left(t-T_{0}\right)} \sum_{j=1}^{n}\left|b_{i j}\right|_{L^{p}\left(T_{0}, T\right)}\left|u_{j}\right|_{\tau}+\left|f_{i}\right|_{L^{1}\left(T_{0}, T\right)}
$$

It follows that

$$
\left|\Gamma_{i}(u)(s)\right|_{X_{i}} \leq \sum_{j=1}^{n} \widetilde{m_{i j}}\left|u_{j}\right|_{\tau} e^{\theta\left(t-T_{0}\right)}+\Lambda_{i} \quad \text { for } s \in[t-\tau, t],
$$

where

$$
\begin{gathered}
\widetilde{m_{i j}}=m_{i j}+\frac{1}{\theta(q \theta)^{1 / q}}\left|a_{i j}\right|_{L^{1}\left(0, T-T_{0}\right)}+\frac{1}{\theta}\left|b_{i j}\right|_{L^{p}\left(T_{0}, T\right)}, \\
\Lambda_{i}=\lambda_{i}+\left|f_{i}\right|_{L^{1}(0, T)}+\left(T-T_{0}\right)\left|d_{i}\right|_{L^{1}\left(0, T-T_{0}\right)} .
\end{gathered}
$$

This gives the estimate

$$
\left|\Gamma_{i}(u)\right|_{C_{\theta}\left(\left[T_{0}-\tau, T\right], X_{i}\right)} \leq \sum_{j=1}^{n} \widetilde{m_{i j}} R_{j}+\Lambda_{i} .
$$

Also taking into account (3.13), we may conclude that

$$
\left|\Phi_{i}(u)+\Psi_{i}(v)\right|_{\tau}=\left|\Gamma_{i}(u)\right|_{\tau} \leq \sum_{j=1}^{n} \widetilde{m_{i j}} R_{j}+\Lambda_{i} \quad \text { for } i \in I_{1} .
$$

Since for $i \in I_{2}$, the structure of $\Phi_{i}(u)+\Psi_{i}(v)$ is analogue to that of $\Gamma_{i}$, we easily see that we also have

$$
\left|\Phi_{i}(u)+\Psi_{i}(v)\right|_{\tau} \leq \sum_{j=1}^{n} \widetilde{m_{i j}} R_{j}+\Lambda_{i} \quad \text { for } \quad i \in I_{2} .
$$


Hence (3.4) holds with $\widetilde{M}=\left[\widetilde{m_{i j}}\right]$ and $\Lambda=\left(\Lambda_{1}, \ldots, \Lambda_{n}\right)^{t r}$. Clearly, the matrix $\widetilde{M}$ is close enough to $M$ if $\theta$ is sufficiently large.

Proof of Lemma 3.3. Similar estimations to those in the proof of Lemma 3.2 give for $i \in I_{1}$ and any $u, v \in C([-\tau, T], X)$,

$$
\left|\Gamma_{i}(u)-\Gamma_{i}(v)\right|_{C\left(\left[-\tau, T_{0}\right], X_{i}\right)} \leq \sum_{j=1}^{n} m_{i j}\left|u_{j}-v_{j}\right|_{C\left(\left[-\tau, T_{0}\right], X_{j}\right)}
$$

and

$$
\left|\Gamma_{i}(u)-\Gamma_{i}(v)\right|_{C_{\theta}\left(\left[T_{0}-\tau, T\right], X_{i}\right)} \leq \sum_{j=1}^{n} \widetilde{m}_{i j}\left|u_{j}-v_{j}\right|_{\tau}
$$

Hence

$$
\left|\Gamma_{i}(u)-\Gamma_{i}(v)\right|_{\tau} \leq \sum_{j=1}^{n} \widetilde{m}_{i j}\left|u_{j}-v_{j}\right|_{\tau} \quad\left(i \in I_{1}\right) .
$$

For $i \in I_{2}$, from $\left(\mathrm{H}_{3}\right)$, we obtain

$$
\left|\Phi_{i}(u)-\Phi_{i}(v)\right|_{\tau} \leq \sum_{j=1}^{n} c_{i j}\left|u_{j}-v_{j}\right|_{\tau} \quad\left(i \in I_{2}\right) .
$$

Consequently,

$$
\|\Phi(u)-\Phi(v)\| \leq \widehat{M}\|u-v\|
$$

where $\widehat{M}$ is the $n \times n$ square matrix $\left[\widehat{m}_{i j}\right]$, with

$$
\widehat{m}_{i j}= \begin{cases}\widetilde{m}_{i j} & \text { for } i \in I_{1}, j \in I \\ c_{i j} & \text { for } i \in I_{2}, j \in I .\end{cases}
$$

Clearly $\widehat{M} \leq \widetilde{M}$, hence according to Proposition 2.3 , the spectral radius of $\widehat{M}$ is less than one. Then (3.14) shows that $\Phi$ is a generalized contraction in Perov's sense.

Proof of Lemma 3.4. The first components of $\Psi$ for $i \in I_{1}$ are zero, so compact. The growth conditions for $F_{i}$ and $K_{i}\left(i \in I_{2}\right)$ and the boundedness of $D$ guarantee the uniform integrability of the set $\left\{\Psi_{i}(u): u \in D\right\}$. Since in addition for $i \in I_{2}$, the semigroups generated by $A_{i}$ are compact, we may apply the compactness criterion from Lemma 2.4 to conclude that the operator $\Psi_{i}$ is compact on $D$ for every $i \in I_{2}$.

Remark 3.5. It is useful to analyze the elements of the matrix $M$ to conclude about the contributions of the nonlinear terms to the sufficient condition for the existence of solutions. They show that $b_{i j}(t)$ can be however large for $T_{0}<t \leq T$. The same happens for $a_{i j}(t)(t \in[0, T])$ and $b_{i j}(t)\left(t \in\left[0, T_{0}\right]\right)$ provided that $T_{0}$ is sufficiently small. Also note the special contribution of $\bar{a}_{i j}$ in connection with the "convolution type" integral term of problem (1.1), which is null if $T_{0}=0$ or $T_{0}=T$.

We conclude by two examples illustrating our main result.

Example 3.6. Consider the semilinear integrodifferential equation

$$
\frac{\partial}{\partial t} u(t, x)-\Delta u(t, x)=\int_{0}^{t} \kappa(t-s, u(s, x)) d s+\mu(t) u(t-\tau, x), \quad t \in[0, T], x \in \Omega,
$$


subject to the Dirichlet condition $u(t, x)=0$ for $x \in \partial \Omega$, and to the nonlocal initial condition

$$
u(t, x)=\lambda u(t+T, x), \quad \text { for } \quad x \in \Omega, t \in[-\tau, 0] .
$$

Here $\Omega \subset \mathbb{R}^{N}$ is a smooth bounded domain, $\tau \geq 0,0<\lambda<1, \kappa:[0, T] \times \mathbb{R} \rightarrow \mathbb{R}$ and $\mu:[0, T] \rightarrow \mathbb{R}$ are continuous functions. The problem is of type (1.1), where $n=m=1, X=L^{2}(\Omega), A=-\Delta, D(A)=H^{2}(\Omega) \cap H_{0}^{1}(\Omega), K, F, \alpha$ are defined as follows:

$$
\begin{aligned}
K, F & : \quad[0, T] \times C\left([-\tau, 0], L^{2}(\Omega)\right) \rightarrow L^{2}(\Omega), \\
K(t, v) & =\kappa(t, v(0)), \quad v \in C\left[[-\tau, 0], L^{2}(\Omega)\right] \\
F(t, v) & =\mu(t) v(-\tau) ; \\
\alpha: C\left([-\tau, T], L^{2}(\Omega)\right) & \rightarrow C\left([-\tau, 0], L^{2}(\Omega)\right), \quad \alpha(v)(t)=\lambda v(t+T) .
\end{aligned}
$$

It is clear that $T_{0}=T$ and $\left(H_{2}\right)$ and $\left(H_{3}\right)$ hold with $b_{11}(t)=\mu(t)$ and $c_{11}=\lambda$. Also $\left(H_{1}\right)$ holds if there is a function $\gamma \in C\left([0, T], \mathbb{R}_{+}\right)$such that

$$
|\kappa(t, y)-\kappa(t, z)| \leq \gamma(t)|y-z| \quad \text { for all } t \in[0, T] \text { and } y, z \in \mathbb{R} \text {. }
$$

It is easy to check that $a_{11}(t)=\gamma(t)$. Also $\bar{a}_{11}=0$. Therefore, Theorem 3.1 yields the following conclusion: If

$$
T|\gamma|_{L^{1}(0, T)}+|\mu|_{L^{1}(0, T)}<1-\lambda,
$$

then the problem has a unique mild solution $u \in C\left([-\tau, T], L^{2}(\Omega)\right)$.

Example 3.7. Let us consider a semilinear reaction-diffusion integrodifferential system with Neumann boundary conditions and multi-point nonlocal initial conditions

$$
\left\{\begin{array}{lr}
\frac{\partial u}{\partial t}(t, x)-\varkappa_{1} \Delta u(t, x)=\int_{0}^{t} \kappa_{1}(t-s, u(s, x)) d s-\lambda_{1} u(t, x)+\mu_{1}(t) v(t-\tau, x), & \text { in } Q \\
\frac{\partial v}{\partial t}(t, x)-\varkappa_{2} \Delta v(t, x)=\int_{0}^{t} \kappa_{2}(t-s, v(s, x)) d s+\mu_{2}(t) u(t-\tau, x)-\lambda_{2} v(t, x), & \text { in } Q, \\
\frac{\partial}{\partial \nu} u(t, x)=\frac{\partial}{\partial \nu} v(t, x)=0, & \text { on } \Sigma, \\
u(t, x)=\varphi(t)(x)+\sum_{k=1}^{p_{1}} \beta_{1 k} u\left(t_{1 k}+t, x\right), & \text { in } Q_{\tau}, \\
v(t, x)=\psi(t)(x)+\sum_{k=1}^{p_{2}} \beta_{2 k} v\left(t_{2 k}+t, x\right), & \text { in } Q_{\tau},
\end{array}\right.
$$

where $Q=[0, T] \times \Omega, \Sigma=[0, T] \times \partial \Omega, Q_{\tau}=[-\tau, 0] \times \Omega, \Omega \subset R^{N}$ is a smooth bounded domain, $\varkappa_{1}, \varkappa_{2}, \lambda_{1}, \lambda_{2}>0, \tau \geq 0$ and $0<t_{i 1}<\ldots<t_{i p_{i}} \leq T$ for $i=1,2$. We assume that $\kappa_{1}, \kappa_{2}:[0, T] \times \mathbb{R} \rightarrow R$ are continuous; $\varphi, \psi \in C\left([-\tau, 0], L^{2}(\Omega)\right)$, and $\mu_{i} \in C\left([0, T] ; \mathbb{R}_{+}\right), i=1,2$. 
We apply Theorem 3.1 with $X_{1}=X_{2}=L^{2}(\Omega)$, and to the operators $A_{i}: D\left(A_{i}\right) \rightarrow$ $L^{2}(\Omega)(i=1,2)$ given by

$$
\begin{aligned}
D\left(A_{i}\right) & =\left\{u \in H^{2}(\Omega): \frac{\partial u}{\partial \nu}=0 \text { on } \partial \Omega\right\}, \\
A_{i} u & =\varkappa_{i} \Delta u-\lambda_{i} u,
\end{aligned}
$$

which generate compact semigroups [9, Theorem 1.11.8].

Here $I_{1}=\emptyset$ and $I_{2}=I=\{1,2\}$,

$$
\begin{gathered}
c_{i i}=\sum_{k=1}^{p_{i}}\left|\beta_{i k}\right|(i=1,2), \\
c_{12}=c_{21}=0, b_{11}=b_{22}=0
\end{gathered}
$$

and

$$
b_{12}(t)=\mu_{1}(t), b_{21}(t)=\mu_{2}(t) .
$$

Also $T_{0}=\max \left\{t_{i j}: j=1, \ldots, p_{i} ; i=1,2\right\}$.

Assume that the functions $\kappa_{1}$ and $\kappa_{2}$ are bounded, i.e.,

$$
\left|\kappa_{i}(t, y)\right| \leq d_{i}, \quad i=1,2, \text { for all } t \in[0, T] \text { and } y \in \mathbb{R} .
$$

Then $a_{i j}=0$ for $i, j=1,2$. Therefore, according to Theorem 3.1, if the spectral radius of the matrix

$$
M=\left[\begin{array}{cc}
\sum_{k=1}^{p_{1}}\left|\beta_{1 k}\right| & \left|\mu_{1}\right|_{L^{1}\left(0, T_{0}\right)} \\
\left|\mu_{2}\right|_{L^{1}\left(0, T_{0}\right)} & \sum_{k=1}^{p_{2}}\left|\beta_{2 k}\right|
\end{array}\right]
$$

is less than one, then the problem (3.15) has at least one mild solution in $C([-\tau, T]$, $\left.L^{2}(\Omega) \times L^{2}(\Omega)\right)$.

Acknowledgements. The work of Sylvain Koumla was supported by a grant from the University Agency of the Francophonie (AUF) in relation to the Romanian National Authority for Scientific Research as part of "Eugen Ionescu" Postdoctoral Fellowship Programme 2017-2018.

\section{References}

[1] Baras, P., Hassan, J.C., Veron, L., Compacité de l'opérateur définissant la solution d'une équation d'évolution non homogène, C.R. Acad. Sci. Paris 284(1977), 779-802.

[2] Bolojan-Nica, O., Infante, G., Precup, R., Existence results for systems with coupled nonlocal initial conditions, Nonlinear Anal., 94(2014), 231-242.

[3] Bolojan, O., Infante, G., Precup, R., Existence results for systems with coupled nonlocal nonlinear initial conditions, Math. Bohem., 140(2015), 371-384.

[4] Bolojan, O., Precup, R., Semilinear evolution systems with nonlinear constraints, Fixed Point Theory, 17(2016), 275-288.

[5] Bolojan, O., Precup, R., Hybrid delay evolution systems with nonlinear constraints, Dynam. Systems Appl., 27(2018), 773-790.

[6] Boucherif, A., Akca, H., Nonlocal Cauchy problems for semilinear evolution equations, Dynam. Systems Appl., 11(2002), 415-420. 
[7] Boucherif, A., Precup, R., On the nonlocal initial value problem for first order differential equations, Fixed Point Theory, 4(2003), 205-212.

[8] Boucherif, A., Precup, R., Semilinear evolution equations with nonlocal initial conditions, Dynam. Systems Appl., 16(2007), 507-516.

[9] Burlică, M.D., Necula, M., Roşu, D., Vrabie, I.I., Delay Differential Evolutions Subjected to Nonlocal Initial Conditions, Chapman and Hall/CRC Press, 2016.

[10] Burlică, M., Roşu, D., Vrabie, I.I., Abstract reaction-diffusion systems with nonlocal initial conditions, Nonlinear Anal., 94(2014), 107-119.

[11] Byszewski, L., Theorems about the existence and uniqueness of solutions of semilinear evolution nonlocal Cauchy problems, J. Math. Anal. Appl., 162(1991), 494-505.

[12] Cardinali, T., Precup, R., Rubbioni, P., A unified existence theory for evolution equations and systems under nonlocal conditions, J. Math. Anal. Appl., 432(2015), 1039-1057.

[13] Cazenave, T., Haraux, A., An Introduction to Semilinear Evolution Equations, Oxford University Press, New York, 1998.

[14] Chabrowski, J., On nonlocal problems for parabolic equations, Nagoya Math. J., 93(1984), 109-131.

[15] Cioranescu, N., Sur les conditions linéaires dans l'intégration des équations différentielles ordinaires, Math. Z., 35(1932), 601-608.

[16] Conti, R., Recent trends in the theory of boundary value problems for ordinary differential equations, Boll. Un. Mat. Ital., 22(1967), 135-178.

[17] García-Falset, J., Reich, S., Integral solutions to a class of nonlocal evolution equations, Commun. Contemp. Math., 12(2010), 1032-1054.

[18] Goldstein, J.A., Semigroups of Linear Operators and Applications, Oxford University Press, New York, 1985.

[19] Infante, G., Maciejewski, M., Multiple positive solutions of parabolic systems with nonlinear, nonlocal initial conditions, J. London Math. Soc., 94(2016), 859-882.

[20] Jackson, D., Existence and uniqueness of solutions to semilinear nonlocal parabolic equations, J. Math. Anal. Appl., 172(1993), 256-265.

[21] Karakostas, G.L., Tsamatos, P.Ch., Existence of multiple positive solutions for a nonlocal boundary value problem, Topol. Methods Nonlinear Anal., 19(2002), 109-121.

[22] Kerefov, A.A., Nonlocal boundary value problems for parabolic equations, (Russian), Differ. Uravn., 15(1979), 74-78.

[23] Kolmanovskii, V., Myshkis, A., Applied Theory of Functional Differential Equations, Kluwer, Dordrecht, 1992.

[24] Lin, Y., Liu, J.H., Semilinear integrodifferential equations with nonlocal Cauchy problem, Nonlinear Anal., 26(1996), 1023-1033.

[25] Liu, J.H., A remark on the mild solutions of non-local evolution equations, Semigroup Forum, 66(2003), 63-67.

[26] McKibben, M., Discovering Evolution Equations with Applications, Vol. I, Chapman \& Hall/CRC, 2011.

[27] Necula, M., Vrabie, I.I., Nonlinear delay evolution inclusions with general nonlocal initial conditions, Ann. Acad. Rom. Sci. Ser. Math., 7(2015), 67-97.

[28] Nica, O., Initial-value problems for first-order differential systems with general nonlocal conditions, Electron. J. Differential Equations, 2012(2012), no. 74, 1-15. 
[29] Nica, O., Precup, R., On the nonlocal initial value problem for first order differential systems, Stud. Univ. Babeş-Bolyai Math., 56(2011), no. 3, 125-137.

[30] Ntouyas, S.K., Tsamatos, P.Ch., Global existence for semilinear evolution equations with nonlocal conditions, J. Math. Anal. Appl., 210(1997), 679-687.

[31] Olmstead, W.E., Roberts, C.A., The one-dimensional heat equation with a nonlocal initial condition, Appl. Math. Lett., 10(1997), 89-94.

[32] Paicu, A., Vrabie, I.I., A class of nonlinear evolution equations subjected to nonlocal initial conditions, Nonlinear Anal., 72(2010), 4091-4100.

[33] Pao, C.V., Reaction diffusion equations with nonlocal boundary and nonlocal initial conditions, J. Math. Anal. Appl., 195(1995), 702-718.

[34] Precup, R., The role of matrices that are convergent to zero in the study of semilinear operator systems, Math. Comp. Modelling, 49(2009), 703-708.

[35] Štikonas, A., A survey on stationary problems, Green's functions and spectrum of SturmLiouville problem with nonlocal boundary conditions, Nonlinear Anal. Model. Control, 19(2014), 301-334.

[36] Vabishchevich, P.N., Non-local parabolic problems and the inverse heat-conduction problem, (Russian), Differ. Uravn., 17(1981), 761-765.

[37] Viorel, A., Contributions to the Study of Nonlinear Evolution Equations, Ph.D. Thesis, Cluj-Napoca, 2011.

[38] Vrabie, I.I., $C_{0}$-Semigroups and Applications, Elsevier, Amsterdam, 2003.

[39] Vrabie, I.I., Global solutions for nonlinear delay evolution inclusions with nonlocal initial conditions, Set-Valued Var. Anal., 20(2012), 477-497.

[40] Webb, G.F., An abstract semilinear Volterra integrodifferential equation, Proc. Amer. Math. Soc., 69(1978), 255-260.

[41] Webb, J.R.L., Infante, G., Positive solutions of nonlocal initial boundary value problems involving integral conditions, NoDEA Nonlinear Differential Equations Appl., 15(2008), 45-67.

[42] Whyburn, W.M., Differential equations with general boundary conditions, Bull. Amer. Math. Soc., 48(1942), 692-704.

Sylvain Koumla

Département de Mathématiques,

Faculté des Sciences et Techniques,

Université Adam Barka,

B.P. 1173, Abéché, Chad

e-mail: skoumla@gmail.com

Radu Precup

Department of Mathematics,

Faculty of Mathematics and Computer Science,

Babeş-Bolyai University,

400084 Cluj-Napoca, Romania

e-mail: r.precup@math. ubbcluj.ro 